\title{
FATORES RELACIONADOS À FALHA NA EXTUBAÇÃO EM UMA UNIDADE DE TERAPIA INTENSIVA DA AMAZONIA OCIDENTAL BRASILEIRA
}

\author{
Katiuscia Larsen de Abreu Aguiar, Maurien Murielle \\ Barbosa Mendonça, Patrícia Rezende do Prado, Tatiana Lameira Maciel Amaral e \\ Simone Aparecida Fernandes
}

\begin{abstract}
RESUMO
Introdução: A falha da extubação está relacionada a um elevado índice de mortalidade hospitalar. Os fatores relacionados ao insucesso da extubação ajudam na elaboração de um protocolo para conduzir as práticas relacionadas a este processo, atenuando as consequências indesejáveis decorrentes deste desfecho. Objetivo: Identificar as características e desfechos na falha da extubação em pacientes internados sob ventilação mecânica em uma Unidade de Terapia Intensiva (UTI) da Amazônia ocidental brasileira. Método: Estudo descritivo do tipo transversal desenvolvido na UTI Adulto de um hospital de urgência e emergência de Rio Branco, Acre, no período de maio a novembro de 2015. Resultados: A amostra final foi composta por 36 pacientes em processo de extubação, com idade média de 49,03 anos $( \pm 20,73)$, o diagnóstico mais prevalente foi o trauma, com $30,6 \%$, tempo médio de internação na unidade de terapia intensiva de 7 dias e a prevalência de falha na extubação foi de $33,3 \%$. As características clinicas evidenciadas como precursoras da reintubação foram: baixa saturação $(38,9 \%)$, seguido de taquipneia $(27,8 \%)$ e alteração no nível de consciência $(16,7 \%)$. Nenhum protocolo foi utilizado para o desmame. Conclusão: Este estudo evidenciou as características do paciente que está sendo extubado, assim como os fatores relacionados ao seu insucesso, no entanto, ênfase maior pode ser dada à necessidade de utilizar um protocolo para a extubação deste paciente. Este foi um estudo preliminar que instiga novos estudos nesta unidade com o objetivo de identificar relações causais para as dificuldades vivenciadas e oferecer uma assistência direcionada aos pacientes críticos.
\end{abstract}

Palavras-Chave: Unidades de Terapia Intensiva. Respiração Artificial. Extubação. Desmame do Respirador. 


\section{Introdução}

A maioria dos pacientes internados na unidade de terapia intensiva (UTI) utiliza a ventilação mecânica invasiva VMI) como suporte à vida e em algum momento, passará pelo processo de desmame compreendido como a passagem da ventilação artificial para a respiração espontânea seguido da extubaçã1,2,3

Este período é compreendido como desafiador para a equipe multiprofissional da UTI e ocupa mais de $40 \%$ do tempo total da ventilação mecânica invasiva3,4. A retirada precoce ou tardia implica em complicações respiratórias irreversíveis que influenciam negativamente na retirada da via aérea artificial levando ao óbito3,5,6.

A interrupção do suporte ventilatório invasivo deve iniciar quando há melhora do quadro clinico da doença de base3,7. Para que ocorra a descontinuação da VMI é importante a avaliação das condições clínicas do paciente diminuição dos parâmetros do ventilador de forma gradual até que o paciente assuma sua independência ventilatória podendo ser definido como o momento adequado para iniciar a retirada da via aérea artificial, ou seja, a extubação seguida de oferta de oxigênio suplementar8,9.

Ainda que estejam atreladas, a falha do desmame e a falha da extubação não necessariamente tem a mesma causa e consequência. A falha no desmame está relacionado a capacidade dos músculos respiratórios em suportar carga e os estímulos impostos pela ventilação espontânea3. Enquanto a falha da extubação está ligada as complicações de vias aéreas superiores, como laringoespasmo, aumento das secreções e tosse ineficaz, fatores associados ao risco de óbito1,10.

Nos estudos atuais, a forma mais utilizada para a retirada da ventilação mecânica consiste em julgamentos empíricos subjetivos e individualizados6,11. Por isso, a utilização de protocolos baseados em evidência pode oferecer um suporte à equipe multiprofissional na condução dos cuidados críticos que os pacientes internados na UTI requerem6,9,11,12 Embora alguns estudos evidenciem pequenas mudanças nessa conduta, ainda há outra barreira a ser ultrapassada, talvez a mais árdua, que é a mudança das práticas e condutas médicas 1,11 .

Neste estudo, pretende-se identificar a prevalência da falha na extubação, bem como os fatores relacionados a este desfecho

Metodologia

Trata-se de um estudo descritivo do tipo transversal desenvolvido na UTI-Adulto do Hospital de Urgência e Emergência, em Rio Branco,Acre, no período de maio a julho de 2015.

A pesquisa foi aprovada pelo Comitê de Ética do Hospital das Clínicas do Acre, sob o Certificado de Apresentação para Apreciação Ética (CAAE) n n 47577215.2.0000.5009 e seguiu a resolução da Comissão Nacional de Ética em Pesquisa (CONEP) 466/12. Para participação nesta pesquisa, o responsável pelo paciente expressou o consentimento através da assinatura no Termo de Consentimento Livre e Esclarecido.

Foram incluídos no estudo adultos com idade $\geq 18$ anos, de ambos os gêneros, intubados, sob ventilação mecânica invasiva por pelo menos 2 (dois) dias e ser candidato ao desmame da VMI.

Em virtude da inexistência de um protocolo para esse procedimento nesta unidade, a equipe de fisioterapia definiu como requisito mínimo os seguintes critérios: obter êxito no teste de respiração espontânea (TER) e com pontuação $\geq$ 8 na Escala de Coma de Glasgow (ECG). Após a avaliação do nível de consciência, medido pela ECG, foi realizado 0 TER utilizando um tubo T com suplementação de oxigênio ( $\mathrm{FiO} 2$ máxima de 40\%) durante 30 minutos. A fisioterapeuta membro da equipe e pesquisadora, acompanhou esse processo em todos os pacientes.

Como desfecho atribuiu-se a falha na extubação, consequente à necessidade de reintubação em até 48 horas após a retirada da via aérea artificial. A necessidade de reintubação ficou a critério do médico assistente e os motivos que evaram ao retorno da via aérea artificial foram extraídos dos prontuários dos pacientes.

Nos prontuários foram coletadas informaç̃̃es sócio demográficas e clinicas. Utilizou-se o software Statistical Package for the Social Sciences (SPSS), versão 17.0, para a análise estatística.

As variáveis contínuas foram expressas em médias e desvios-padrão ou em medianas e intervalos interquartis, ao passo que as variáveis categóricas foram expressas em frequência relativa.

Resultados

Foram incluídos no estudo 36 pacientes extubados. A média de idade dos pacientes foi de 49,03 $\pm 20,73$ anos, houve predomínio de pacientes do gênero masculino $(66,7 \%)$ e o insucesso na extubação foi de $33,3 \%$.

O diagnóstico clínico mais comum foi o de traumatismo cranioencefálico (em 30,6\%), acompanhado de distúrbios respiratórios (em 27,8\%) e distúrbios cardiovasculares (em 16,7\%), respectivamente (Tabela 1).

Tabela 1: Características e desfecho da população estudada. Rio Branco, Acre, 2015. (N = 36)

\begin{tabular}{|cl|c|}
\hline Variável & $\%$ \\
\hline \multirow{2}{*}{ Sexo } & Masculino & 66,7 \\
& Feminino & 33,3 \\
\hline Idade & (anos) $\quad$ (média \pm d.p.) & $49,03 \pm$ \\
& & 20,73 \\
\hline Diagnóstico & \\
& Distúrbios & \\
& respiratórios & 27,8 \\
& Distúrbios & 16,7 \\
& cardiovasculares & 30,6 \\
& Traumas & 25,0 \\
\hline & Outros & \\
\hline \multirow{2}{*}{ Desfecho } & Sucesso & 66,7 \\
& Insucesso & 33,3 \\
\hline
\end{tabular}

Dos pacientes que foram reintubados, $38,9 \%$ foram em virtude de apresentarem hipoxemia, $27,8 \%$ devido à presença de taquipneia, 16,7\% devido a alteração no nivel de consciência (Tabela 2). 
Tabela 2: Características clínicas do insucesso. Rio Branco, Acre, 2015.

\begin{tabular}{|l|c|}
\hline Variável & $\%$ \\
\hline Saturação arterial de $02<90 \%$ & 38,9 \\
Taquipneia & 27,8 \\
Alteração do nível de consciência & 16,7 \\
Frequência cardíaca > 140 bpm & 11,1 \\
Sudorese e Agitação & 5,5 \\
\hline
\end{tabular}

Quanto aos parâmetros ventilatórios utilizados no desmame verificou-se que $41,7 \%$ utilizaram a pressão de suporte (PSV), seguido de $33,4 \%$ em ventilação mandatória intermitente sincronizada (SIMV). Com relação ao tempo de internação e tempo de ventilação nota-se que a permanência foi de até 7 dias $(77,8 \%)$ (Tabela 3$)$. 0 teste de respiração espontânea foi realizado em todos os pacientes.

Tabela 3: Parâmetros ventilatórios da população estudada. Rio Branco, Acre, 2015.

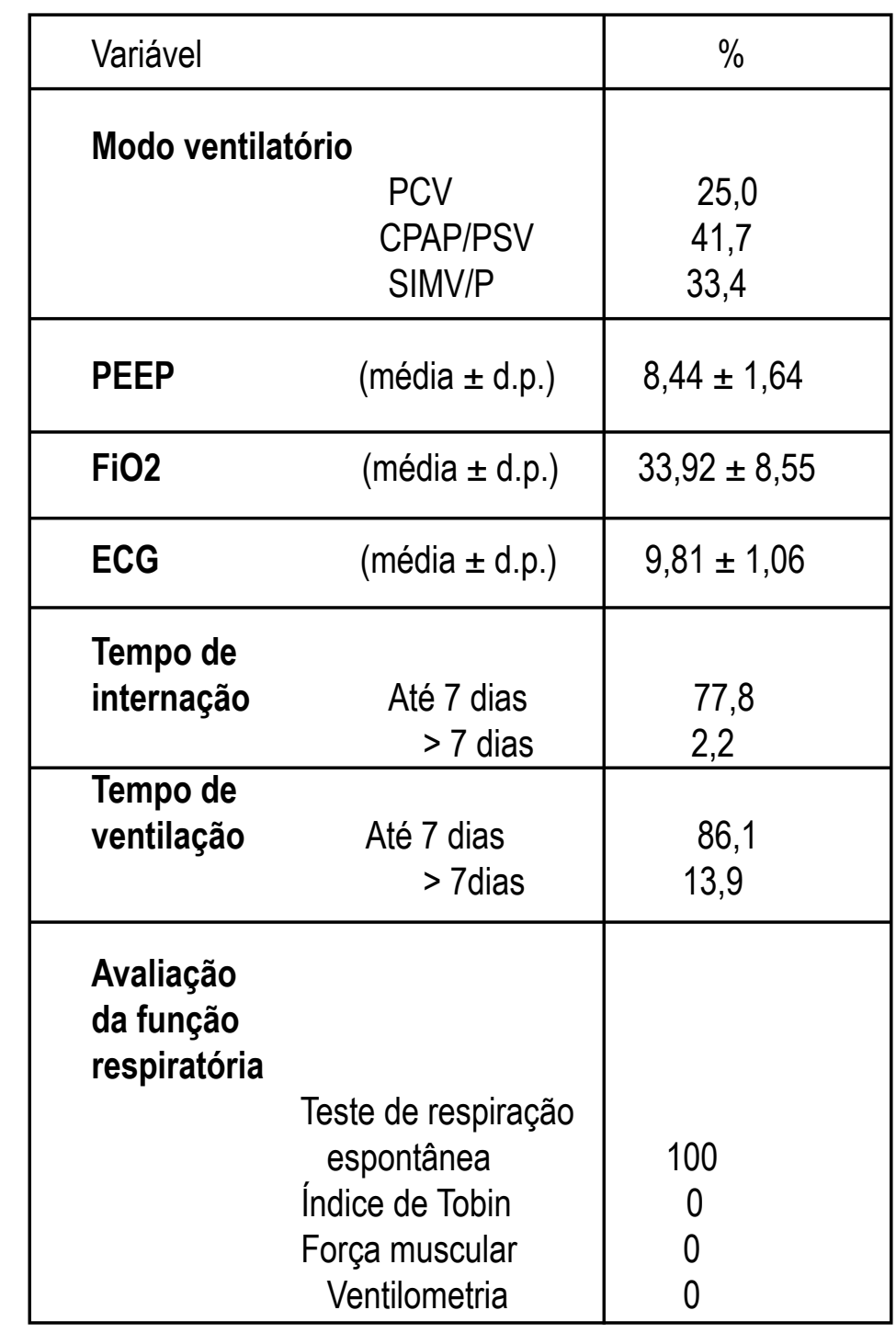

Um estudo clínico prospectivo multicêntrico de coorte realizado em três UTI's de Porto Alegre, no qual comparam dois grupos de pacientes em relação à extubação da VMI, sendo o primeiro extubados utilizando protocolo e o segundo, sem a utilização do mesmo, foi identificado sucesso em $86,7 \%$ dos pacientes que foram extubados sob respaldo de um protocolo, contra $69,6 \%$ dos que não utilizaram um protocolo. Este resultado evidencia dois fatores importantes, ou seja, a utilização de protocolo favorece o sucesso da extubação, e segundo, esta prevalência de sucesso foi maior em relação a nossa pesquisa realizada na Amazônia, demonstrando que é preciso melhorar o processo (sucesso 66,7\%). Neste sentido, ressalta-se a necessidade da extubação ser realizada por um protocolo1.

Este estudo é o primeiro a avaliar as características e desfechos relacionados a falha da extubação em um estado da Amazônia ocidental brasileira e, consequentemente a isso, há uma preocupação com as consequências advindas da extubação mal sucedida. Porém, a realização de estudos em unidades de terapia intensiva com a finalidade de evidenciar a adesão de protocolos baseados em evidencia para desmame e extubação ainda é baixa, observa-se que essa adesão não chega a 1\% nas UTI's e quando são utilizados, os resultados são positivos6,10.

No presente estudo observou-se que o trauma é o diagnóstico com maior frequência entre os pacientes internados nesta unidade, devido ao fato de ser o único hospital de referencia para os casos de emergência de todo o Estado. Isso

sugere a relação com o tempo de internação, o qual, em sua maioria, é de até 7 dias. Essa média de permanência é considerada baixa frente a outros estudos onde o tempo médio é de 16 dias 15.

A assistência ventilatória ao paciente grave tem por objetivo diminuir o trabalho muscular respiratório e manter as trocas gasosas, deixando que o paciente recupere o seu estado fisiológico normal16. Devido as características dos pacientes desta unidade de investigação, a maior prevalência de reintubação está relacionada com a dificuldade de manter os níveis adequados de $\mathrm{O} 2$ e $\mathrm{CO} 2$ no sangue. Sabe-se que os distúrbios relacionados ao trauma deixam o paciente hemodinamicamente instável, podendo causar sequelas permanentes e ou temporárias, com vários graus de comprometimentos e mais suscetível ao óbito17.

A utilização de técnicas e parâmetros para iniciar o processo de desmame da ventilação mecânica invasiva é undamental para atingir exito na etapa seguinte, a extubaccão. Ao entender que o processo de desmame é gradual, às vezes difícil, mas de grande importância para se reestabelecer as condições fisiológicas do paciente. Neste sentido, enfatiza-se o quanto é importante o estabelecimento de condutas e critérios para que haja sucesso nesta etapa de treinamento muscular respiratório3,7,8.

Assim, os métodos utilizados nesta fase devem ter como função principal o treinamento muscular dos músculos respiratórios sem que ocorra a fadiga. O modo PSV, utilizado nesta unidade, e em outros estudos para fase de desmame tem como vantagem o controle sobre o que esta sendo ofertado, ou seja, oferece suporte assistido por pressão, limitando o fluxo em que são predeterminados níveis de pressão positiva na fase inspiratória1,18,19.

Na ventilação mandatória intermitente sincronizada (SIMV) pode-se combinar os modos assistidos e espontâneos porem controlados. Sua utilização não e a mais recomendada, tendo em vista a facilidade de desencadear a fadiga muscular respiratória e com isso aumentar a estadia do paciente na unidade de terapia intensiva em uso de ventilação mecânica invasiva3,20,21.

O objetivo desta pesquisa não foi avaliar o processo de desmame, visto que não seria possível por não haver nenhum protocolo para esta finalidade. No entanto, cabe destacar que o processo de desmame é tão importante quanto o banho no leito, constitui-se como ação diária, onde requer avaliação de critérios

pré-estabelecidos pela equipe multiprofissional e a necessidade de um despertar diário visando o retorno às funções fisiológicas e alta do paciente6,7,18. 
Este estudo evidencia que a falha da extubação decorre, principalmente, do desequilíbrio entre oferta e consumo de oxigênio, gerando hipoxemia levando o paciente a retornar para a VMI. Pode-se ainda sugerir que a inexistência de um protocolo e treinamento adequado na fase do desmame com o paciente esteja gerando a falha na extubação, representado pelos $33,3 \%$ que não tiveram êxito na retirada definitiva da via aérea artificial. Essas inquietações instigam novos estudos nesta unidade, na busca para oferecer serviços de mais qualidade ao paciente crítico.

Ainda, como resultado da reintubação, a razão evidenciada neste estudo foi a ocorrência de taquipneia seguida da alteração do nível de consciência. Estas alterações podem estar relacionadas com a incapacidade dos músculos respiratórios em não conseguirem sustentar a carga imposta pela mecânica ventilatória, evidenciando a fadiga da musculatura.

Conclusão

Este estudo apresentou algumas limitações como não ter estudos anteriores para realizar comparações, porém constitui o passo inicial para melhorar seu processo e realizar futuras pesquisas.

Destaca-se que nesta unidade não há protocolo de extubação, portanto a decisão foi feita pelo médico assistente. Apesar das limitações, o estudo foi conduzido com rigor e dedicação com o objetivo de auxiliar, aprimorar e publicar os resultados encontrados para que novas pesquisas possam ser realizadas.

A elaboração e implementação de um protocolo para o acompanhamento do desmame ventilatório e da extubação é imprescindível. Ressalta-se a importância de definir técnicas e condutas específicas, suprimindo a avaliação subjetiva perpetrada atualmente, sendo possível, assim, reduzir as consequências indesejáveis advindas da reintubação precoce.

\section{REFERÊNCIAS BIBLIOGRÁFICAS}

1. Teixeira C, Maccari JG, Vieira SRR, Oliveira RP, Savi A, Machado AS, et al. Impact of a mechanica ventilation weaning protocol on the extubation failure rate in difficult-to-wean patients. J Bras Pneumol. 2012;38(3):364-71.

2. Azevedo Muniz Y, Braide ASG, de Moraes MCS, Maciera CL, Brito MSR, Viana MCC. Estratégias de desmame da ventilação mecânica em uma Unidade de Terapia Intensiva. ASSOBRAFIR Ciênc. 2015;6(1):31-9.

3. Goldwasser R, Farias A, Freitas EE, Saddy F, Amado V, Okamoto VN. Mechanical ventilation of weaning interruption. Rev Bras Ter Intensiva. 2007;19(3):384-92.

4. Meade M, Guyatt G, Cook D, Griffith L, SinuffT, Kergl C, et al. Predicting success in weaning from mechanical ventilation. CHEST J. 2001;120(6_suppl):400S - 424S.

5. Esteban A, Anzueto A, Alía I, Gordo F, Apezteguía C, Pálizas F, et al. How Is Mechanical Ventilation Employed in the Intensive Care Unit? Am J Respir Crit Care Med. 2000;161(5):1450-8.

6. Goldwasser RS, David CM. Desmame da ventilação mecânica: promova uma estratégia. Rev Bras Ter Intensiva. 2010;19(1):107-12.

7. Carvalho CRR, Toufen Junior C, Franca SA. Ventilação mecânica: princípios, análise gráfica e modalidades ventilatórias. J Bras Pneumol. 2007;33:54-70.
8. Gonçalves EC, Silva EC, Basile Filho A, Auxiliadora-Martins M, Nicolini EA, Gastaldi AC. Low pressure support changes the rapid shallow breathing index (RSBI) in critically ill patients on mechanical ventilation. Braz J Phys Ther. 2012;16(5):368-74.

9.ColomboT, Boldrini AF, Juliano SRR, Juliano MCR, Houly JGS, Gebara OCE, et al.Implementation, assessment and comparison of the T-Tube and pressure-support weaning protocols applied to the intensive care unit

patients who had received mechanical ventilation for more than 48 hours. Rev Bras Ter Intensiva. 2007;19(1):31-7.

10. Paredes ER, Junior VN, de Oliveira ACT. Protocolo de prevenção de falha de extubação como estratégia para evitar as complicações da reintubação precoce. UNILUS Ensino E Pesqui. 2013;10(19):12-9.

11. José A, Pasquero RC, Timbó SR, Carvalhaes SRF, dos Santos Bien U, Dal Corso S. Efeitos da fisioterapia no desmame da ventilação mecânica. Fisioter Em Mov [Internet]. 2013 [citado 15 de agosto de 2016];26(2). Available at: http://www2.pucpr.br/reol/index.php/RFM?dd1=7807\&dd99=pdf

12. Nemer SN, Barbas CSV. Predictive parameters for weaning from mechanical ventilation. J Bras Pneumol. 2011;37(5):669-79.

13. Pinto WAM, Rossetti HB, Araújo A, Júnior S, Jonas J, Salomão H, et al. Impact of a continuous education program on the quality of assistance offered by intensive care physiotherapy. Rev Bras Ter Intensiva. 2014:26(1):7-13.

14. Cubo E. Perfil dos fisioterapeutas que trabalham em terapia intensiva na cidade do recife e região metropolitana [Internet]. BJPT. [citado 8 de março de 2016]. Available at: http://www.rbf-bjpt.org.br/ article/54d9fbc85ce02af93c00000c

15. Nogueira $L$ de $S$, Sousa RMC de, Domingues $C$ de $A$. Severity of trauma victims admitted in intensive care units: comparative study among different indexes. Rev Lat Am Enfermagem. 2009;17(6):1037-42.

16. Junior S, Da SHA, Vasconcelos AGG, Griep RH, Rotenberg L. Validity and reliability of the work ability index (WAI) in nurses' work. Cad Saúde Pública. 2011; 27(6):1077-87.

17. de Almeida Gentile JK, Himuro HS, Rojas SSO, Cordeiro V, Veiga LECA, de Carvalho JC. Condutas no paciente com trauma crânioencefálico. Rev Bras Clin Med São Paulo. 2011;9 (1):74-

18. Barbas CSV, Ísola AM, Farias AM de C, Cavalcanti AB, Gama AMC, Duarte ACM, et al. Brazilian recommendations of mechanical ventilation 2013. Part 2. Rev Bras Ter Intensiva. 2014;26 (3):215-39.

19. Pereira PC, Oliveira LHS, Amâncio JS, Moraes FC. Desmame da ventilação mecânica: comparação entre pressão de suporte e tubo T: uma revisão de literatura doi: http://dx. doi. org/10.5892/ruvrv. 2013.111 500511. Rev Universidade Vale Rio Verde. 2013;11(1):500-11.

20. Mascarenhas DM, Mejia DPM. Desmame da Ventilação Mecânica em Adultos: Métodos mais Utilizados na Atualidade. [citado 23 de agosto de 2016]; Available at: http://portalbiocursos.com.br/ohs/data/ docs/27/06_-_Desmame_da_VentilaYYo_MecYnica_em_Adultos_MYtodos_mais_Utilizados_na_ Atualidade.pdf 
21. Moraes MA de, Bonatto RC, Carpi MF, Ricchetti SMQ, Padovani CR, Fioretto JR. Comparação entre ventilação mandatória intermitente e ventilação mandatória intermitente sincronizada com pressão de suporte em crianças. J Pediatr. 2009;85(1):15-20. 